\title{
Carbon Emissions Trading Market Mechanism Design: Perspectives of Legal Economics
}

\author{
Chengli Ren ${ }^{1, *}$ \\ ${ }^{1}$ Institute of Economics, Hunan Agricultural University, Changsha, Hunan Province, China \\ *Corresponding author. Email: renchengli@stu.hunau.edu.cn
}

\begin{abstract}
The national carbon market trading is about to go live. This paper mainly explores the problems and some personal opinions that the carbon emission right market has exposed after ten years of experience in the pilot based on the dual disciplines of law and economics. According to China's national conditions, it puts forward more optimization proposals. It is hoped that the research of this paper can make the market mechanism of carbon emission right trading in China more perfect, to promote the process of greenhouse gas emission control. Carbon market construction will continue to strengthen. We can better cope with climate change and air pollution prevention and control, ecological environment protection, and other work. The characteristic of this paper is that the research is more abundant and rigorous by using the research method of the intersection of law and economics and studying from many perspectives.
\end{abstract}

Keywords: Carbon emission rights, Law and economics, Transaction mechanism design.

\section{INTRODUCTION}

At the Leaders' Climate Summit on April 22, 2021, General Secretary Xi Jinping said that China has decided to accept the Montreal Protocol's Kigali Amendment, strengthen controls on non-CO2 greenhouse gases, and launch a national carbon market online deal. Therefore, in the current situation, establishing a unified national carbon trading market is particularly important. In recent years, the market transaction of carbon emission rights, as a means of dealing with air pollution effectively, has been gradually piloted in China. Since 2011, China has worked in Beijing, Shanghai, Tianjin, Hubei, Shenzhen, Guangdong, Chongqing, and Fujian as pilot cities and has accumulated valuable experience for the national unified carbon emissions trading market. This paper summarizes the previous research and puts forward new suggestions for designing China's carbon emission rights trading market mechanism.

This paper uses the combination of law and economics to study the market mechanism of carbon emissions trading in China. Through the research of this paper, China's carbon emission rights trading market mechanism will be more perfect to promote the process of greenhouse gas emission control. Carbon market construction will continue to strengthen, and we can better combat climate change and air pollution prevention and control, ecological environment protection, and other work

\section{A REVIEW OF LITERATURE}

\subsection{Carbon emissions trading}

Carbon emissions trading means that within a certain area, the total amount of carbon dioxide emissions of greenhouse gases within a specified time limit is determined, distributed to individuals or organizations in the form of quotas or emission permits. Therefore, they have a legitimate right to carbon emissions, and allow such rights to trade among market participants like commodities, to ensure that the actual carbon emissions do not exceed the total amount of limited emissions.

\subsection{Clean development mechanism}

The Clean Development Mechanism (CDM) refers to one of the flexible compliance mechanisms introduced in the Kyoto Protocol. The core element is to allow their Parties, i.e., developed and non-parties or developing countries, to transfer and obtain project-level emission offsets, thereby implementing greenhouse gas reduction projects in developing countries. It is undeniable that China has significantly increased its 
awareness and ability to address climate change in the years since the CDM project was developed, provided valuable experience in the development of China's emission reduction projects, and trained the first batch of technical personnel for China's carbon market.

CDM, after 2012 gradually losing its dominance as China's carbon reduction driver. China's cumulative capacity to cope with climate change through CDM has, in turn, contributed significantly to the design and operation of the domestic carbon market in a relatively short period.

\subsection{Definition of the legal nature of the right to carbon emissions}

At present, the "right to carbon emissions" is not clearly defined in legislation (only in the Interim Measures for the Administration of Carbon Emissions Trading, which states that "the right to carbon emissions refers to the right to emit greenhouse gases into the atmosphere obtained by law"). There is currently no unified view in the academic community on the definition of "right to carbon emissions". Carbon emission rights can be classified as environmental rights and are a fundamental human right [1]. The object of carbon emission rights can be "materialized", which should belong to the concession [2]. The public right to carbon emissions is much more colorful than its private rights. Classifying it as an administrative license right can also avoid the difficulties other doctrines face [3]. The carbon emission right is similar in nature to the "mining right" and "water right" in China's Property Law and can be defined as a "quasi-use beneficial property right" [4]. There are many heresies on the legal attribute of carbon emission rights, and there is no consensus.

\subsection{China's carbon emission rights trading market mechanism design}

A high monopoly of the carbon emissions industry will seriously hinder the construction of China's carbon emissions trading market [5]. China should first develop the spot market for carbon trading before developing the derivative market for carbon trading [6]. The design of the carbon trading mechanism should reduce the total amount of carbon quotas, reduce the distribution of free quotas and increase the price of carbon trading, which will increase carbon emission reduction efforts while increasing the inhibition of the economy [7]. Hebei Province carbon emissions accounted for more than $70 \%$ of the total emissions in the Beijing-Tianjin-Hebei region, proposed to speed up the construction of Hebei Province and even the whole area of Beijing-TianjinHebei carbon trading market, the formulation of carbon financial market development strategy planning, innovative rich and diverse carbon finance products, etc., to reduce emission reduction costs [8]. Joint supervision and enforcement of carbon emissions trading in the Beijing-Tianjin-Hebei region, providing institutional guarantees for regional carbon trading [9]. Unused carbon rights should be allowed to be stored until the next period, which will make the trading mechanism more stable. "My proposal for a total ban on lending is aimed at preventing a vicious circle. The firm entering during the trading phase cannot obtain an initial share of the rationed emissions, so the firm needs to buy in the market."[10]

\subsection{Research review}

To sum up, China's carbon emission rights trading market mechanism has been basically complete research. However, there are still some major issues that have not been deeply studied and implemented, and few people will be economics and law combined to explore. Therefore, this paper will stand in the perspective of French economics, and combined with China's national conditions, for China's carbon emissions trading market and carbon emissions trading management to put forward more complete recommendations.

\section{DESIGN OF THE MARKET TRADING MECHANISM FOR CARBON EMISSION RIGHTS}

The significance of setting up the carbon emission right market trading mechanism is to optimize the allocation of carbon emission space resources through the market mechanism, provide economic incentives for the emission of real carbon emission reduction, and reduce greenhouse gas emissions based on the market mechanism, on the premise of setting mandatory total carbon emission control targets and allowing carbon emission quota trading. The carbon emissions market trading mechanism helps to address carbon emission reduction and total greenhouse gas emissions control and peaks. The design of the carbon emission right market trading mechanism should generally consider the total amount setting and initial distribution, the design of the offset mechanism, the market supervision system, other carbon finance innovations, and so on.

\subsection{Total setup and initial allocation}

A unified quota allocation method can reflect the advanced nature of corporate carbon emissions and emission reduction. By optimizing the total quota to effectively adjust the carbon price, play the role of the market mechanism, improve the enthusiasm of enterprises to participate in the carbon market, promote enterprises to implement more scientific and efficient emission reduction programs. Setting the total emission permit amount is the premise of the operation of the emission rights trading system. 
In the pilot areas of China, to carry out carbon emissions trading work generally take the form of free government distribution to enterprises to distribute the initial quota of carbon emission rights. Specifically, the pilot regions are free of charge to the industry to issue initial quotas for carbon emission rights. There are two criteria: first, historical emissions law; in addition, there is the auction method. The so-called historical emission method, that is, according to the amount of carbon emitted by enterprises in the past a certain period to determine the amount of carbon that can be emitted at present, industry benchmark law. According to the total amount of carbon emissions per unit of products in the enterprise and technical level to determine a relatively advanced emission rate, the emission rate and its total products multiplied, to arrive at the initial quota of carbon emissions of enterprises. However, in theory and in practice, these allocation rules are not without doubt. The 2019-2020 National Carbon Emissions Trading Quota Setting and Distribution Implementation Plan (Starting Point Electricity Industry) document proposes that the provincial ecological environment authorities shall approve the quota quantity of each key emission unit according to the actual output of the key emission units in their administrative areas for 2019-2020 and the quota allocation methods and carbon emission baseline values determined by the scheme. The total quota of each provincial administrative region will be increased, and the total amount of national quota will be determined. The Measures for the Management of Carbon Emissions Trading (Trial) implemented from February 1, 2021, essentially follow the previous proposal to determine the total amount of carbon emissions, considering factors such as economic growth, industrial restructuring, energy structure optimization, and coordinated control of atmospheric pollutant emissions, in accordance with national greenhouse gas emission control requirements.

Overall, the determination of total carbon emissions needs to consider a number of factors to integrate historical emissions, technical level, and in light of the actual situation of the year, timely monitoring of carbon emissions, and flexible adjustment. According to the Measures for the Administration of Carbon Emissions Trading (Trial) and its implementation, priority is given to unpaid distribution. The remaining few are sold in the form of transactions most reliably and effectively.

\subsection{Design of the offset mechanism}

The offset mechanism is to obtain emission reductions by reducing emissions from activities outside the carbon trading system. Offset credits must be rigorously reviewed before they can be issued to ensure the authenticity and extranets of the emissions cuts, which would not have happened. Companies can then purchase these lines of credit to fulfill some of their compliance obligations under the carbon trading system. Typical offset projects include renewable energy, energy conservation and retrofits, waste management, agriculture, and forestry. Because offset credits come from outside the carbon trading system, emissions (i.e., totals) allowed in the carbon trading system are increased. As a result, governments often limit the amount of offset available (such as the number of quotas that companies receive or a percentage of the level of compliance required) to ensure that most emission reductions occur within the industry covered by carbon trading systems. In addition, to maintain the quality of the offset mechanisms used, carbon market policymakers often limit them by project type or source.

It should be noted that for traditional enterprises with high carbon consumption, CCER [11] proposes a solution of "offsetting credit with project carbon", but its landing is not permanent for enterprises. Reviewing the history of the CCER project system, it has attracted attention due to market confusion, irregularities, and other issues. In March 2017, the National Development and Reform Commission (NDRC) issued a notice suspending the processing of applications for voluntary greenhouse gas reduction projects. The Environmental Protection Agency (EDF)also issued in 2020 "Several Expectations for the Future Development of CCER", hoping to speed up the restart of the CCER project as soon as possible to revise and improve the Interim Measures and speed up the issuance of relevant supporting rules. It also simplified the CCER management process, introduce penalties, increase the cost of violations of laws and regulations of various types of market participants, for the future CCER restart pointed out the direction. In the article "The Launch of the International Aviation Carbon Market is Here, CCER Project System Urgently Needs "Breaking Bad "published by EDF in March 2021, it is stated that the CCER Project System suspended the filing of new projects and emission reductions in March 2017, that the filing of new projects and emission reductions under the CCER project system is still pending, and that its own CORSIA qualified carbon emission reduction targets are insufficiently supplied and CORSIA "Potential increase in demand for emission reduction targets and the current status of whether CORSIA will allow eligible carbon reduction targets for the CCER project system to be extended, it is urgent for projects to restart new projects and emission reduction filings, including the development of new methodologies." In addition, the article suggests that "the improvement of systems and systems related to the CCER project system needs to be integrated and coordinated to consider the requirements of the national carbon market and CORSIA." "The CCER project system needs to properly address the accounting of international transfers of carbon reduction targets under the Paris Agreement and avoid duplication of calculations." It's also worth thinking about. 
While it remains to be seen when CCER will restart, some important details related to CCER are emerging as the carbon market continues to heat up. Article 29 of the Measures for the Administration of Carbon Emissions Trading (Trial) (hereinafter referred to as the Pilot Approach) effective February 1, 2021, relates to CCER. At the same time, the pilot approach also qualifies the types of CCERS that are allowed to be used. On March 24, the Beijing Municipal Government issued a notice on the implementation plan for constructing a modern environmental governance system in Beijing. Article 23 clearly states that Beijing will "build a national voluntary greenhouse gas emission reduction management and trading center". On March 30, the Ministry of Ecology issued the Notice on the public consultation on the > Opinions of the Interim Regulations on the Administration of Carbon Emissions Trading in < (Draft Amendment), of which Article 13 is related to CCER. A comparison of the pilot approach with the draft revision reveals that there has been no change in the type of CCER project available. However, the offsetting ratio is not included in the Interim Regulations on the Administration of Carbon Emissions Trading (draft amendment). This is understandable as a higher level of the decree. According to the pilot approach and the revised draft, the types of CCERS allowed in the context of the national carbon market include renewable energy, forestry carbon sinks, and methane use. Renewable energy refers to projects powered and heated by wind, solar, hydro, biomass, geothermal and marine energy. In fact, in the first phase, renewable energy projects have been the largest of all project types, accounting for approximately $70 \%$ of total development. Renewable energy projects are expected to remain the second phase of the largest. Forest carbon sinks are CCER projects developed under the plant carbon sink function. In the first phase, carbon sink projects account for only $3 \%$ of all projects, which is closely related to the complexity of carbon sink project development techniques and the length of development cycles. In the second phase, with the reduction of compliant CCER types, forestry carbon sink projects are likely to become hot spots for CCER development. If there are high-quality forestry carbon sink projects, it will be particularly critical to select an advisory body proficient in CCER development technology.

So, in constructing the next CCER restart project, I think the first point is that CCER transaction access needs to be properly designed. Excessive liberalization of CCER access will impact the carbon quota market, reduce the effectiveness of quotas, reduce the motivation of enterprise endogenous emission reduction, and make full use of CCER's contribution to the carbon market. The second point is to simplify the CCER management process, optimize the penalty mechanism, upgrade the cost of illegal and illegal enterprises, to avoid market confusion, irregularities, and other problems. The third point is that the construction of CCER needs to consider the comprehensive coordination of the national carbon market and CORSIA related requirements so that the CCER project system in the restart, not only to meet the needs of the international aviation carbon market but also to help the healthy and orderly development of the national carbon market.

\subsection{Market regulation}

To further standardize the verification activities of enterprise greenhouse gas emissions reporting in the national carbon emissions trading market, the Ministry of Ecological Environment has issued the Guidelines for the Verification of Enterprise Greenhouse Gas Emissions Reports (Trial) (hereinafter referred to as the Guide) in accordance with the Measures for the Management of Carbon Emissions Trading (Trial). Subsequently, the Ministry of Ecological Environment has publicly solicited opinions on the Interim Regulations on the Administration of Carbon Emissions Trading (Amendment to the Draft) (hereinafter referred to as the "Amendment to the Draft"), with a deadline of April 30, 2021. Regulatory objects mainly include enterprises involved in carbon trading, carbon trading platforms (carbon exchanges or carbon trading companies), intermediary organizations, industry organizations, related financial institutions, etc., to better play the role of market mechanisms, promote the construction of the national carbon market, unified policy planning standards, unified monitoring and evaluation, unified supervision and enforcement, unified accountability of inspectors is particularly important. To ensure accurate data, the Guide also provides a range of institutional safeguards. For example, suppose the key emission unit objects to the verification results after verification. In that case, it may also appeal and review the results, and the review's conclusions shall be made public. According to the Measures for the Administration of Carbon Emissions Trading (Trial), the competent department of the ecological environment at a higher level may also supervise and inspect the verification results.

In my opinion, in addition to building a government supervision system, we should also build a third-party regulatory system that uses exchanges, consumer groups, public opinion, and the media to monitor the operation of the carbon trading market. In addition, internal self-monitoring is necessary.

\subsection{Other Carbon Finance Innovations}

Increasing financial innovation and enriching carbon financial products will help guide more social capital to actively participate in constructing the carbon market. In constructing the carbon trading market, we will 
strengthen the cultivation of carbon finance and encourage financial institutions to participate in carbon market trading. After the national carbon market matures and perfects, we can explore and study the introduction of carbon futures, options, forward products, and other financial derivatives into the carbon market. Then we encourage carbon finance innovation such as quota pledge financing and carbon bonds, and guide financial resources to promote the development of carbon market and the construction of carbon pricing centers with international influence. In relying on the carbon market to promote carbon finance innovation, the National People's Congress representative, the People's Bank of China Guangzhou Branch President Bai Hexiang suggested, learn from the Shanghai-Hong Kong Stock Connect, Shenzhen-Hong Kong Pass, Bond Pass, cross-border financial communication and other domestic and foreign capital interconnection mechanism construction experience, explore the establishment of "carbon market", build and lay the carbon market domestic and foreign trading channels to attract global funds to participate in the transaction. Develop more financial attributes of the carbon market to send a clear signal to society and market players to encourage innovation in carbon finance products. The successful experience of international carbon market financialization has provided some reference for China to explore the development path of carbon finance, and the pilot carbon market in China is also actively expanding its unique business. So, we should further develop the financial attributes of the carbon market and strengthen the innovation and development of financial products in the future. Appropriate relaxation of access standards encourages financial institutions, carbon asset management companies, and other third-party intermediaries to participate in market transactions. We should strengthen overall consideration, systematic planning, clarify the division of responsibilities among various departments, and consider the market access qualifications of non-controlling entities such as relevant financial institutions and carbon asset management companies. Establish a carbon financial risk prevention mechanism and policy system to strengthen carbon financial risk management. At the national strategic level, carbon finance should be integrated into the sustainable development strategy. The legal construction of carbon financial transactions should be improved to prevent carbon financial risks effectively. While encouraging financial institutions to actively participate in carbon finance activities, the management of climate change-related risks should be strengthened, and institutions should be gradually upgraded in their capacity to cope with climate change. Improve the carbon financial trading platform to regulate the risks that may arise from the trading process.

\section{THE CURRENT PROBLEMS IN THE CARBON TRADING MARKET}

\subsection{The system is not perfect.}

Since the National Development and Reform Commission decided to carry out the pilot, the government has promulgated several energy conservation and emission reduction policies. Seven provinces and cities of local regulations or local regulations are also being issued so that the pilot work can be carried out smoothly. However, since local governments formulate these local laws and regulations in accordance with local economic development, they only apply to the situation of each place itself and cannot meet the needs of the national market. Because the level of legislation issued by the policy and regulations is too low, its effectiveness is not enough to coordinate the practical operation between national ministries and between the central and local governments, which will make it difficult to carry out the actual work of various departments and regions.

China should formulate such high-effect superior law as the Carbon Emissions Trading Law, formulate provisions on the verification mechanism, quota allocation, liability for breach of contract and other principles through legislation, provide unified legal principles for the carbon trading market so that all places can be more operational in practice, and avoid loopholes in the law of individual provinces, cities, and enterprises. However, the formulation of a one-line law to take some time. The author believes that it is possible to first issue administrative regulations, in the administrative regulations on carbon emissions of the administrative licensing rights, with these administrative regulations for the development of carbon trading market as temporary legal support, to avoid appearing in practice "cannot be relied upon" situation. To establish a better carbon trading system, China should, in accordance with the theory of sustainable development, adhere to the Constitution and environmental protection law as its superior law, formulate laws and regulations on the trading of carbon emission rights in line with China's national conditions, more specific and detailed practice, clarify the rights and obligations of the government and citizens, enhance the awareness of energy conservation and emission reduction of enterprises, protect the environment and human health. To higher-level legislation, such as the State Council level to ensure the authority of the carbon market, as soon as possible the introduction of the national carbon emissions trading regulations, for the construction of the carbon market system to provide legal support. Based on the Measures for the Administration of Carbon Emissions Trading (Trial), we will further formulate and improve the relevant supporting systems and rules to guide enterprises in carbon trading. 


\subsection{Penalties are too small}

According to the current pilot cities and provisional regulations, the penalties are not strong enough. The authority and responsibility of unclear cause enterprises to look down on compliance. From the figures for excessive fines, the penalties are not large everywhere. Most of the fine amount is under 30,000 yuan, the cost of enterprises over-draining is low. Shanghai imposes a maximum fine of 100,000 yuan, Beijing imposes a penalty of 3 to 5 times the average market price for the over-row portion, and Shenzhen imposes a three-fold fine on the over-queued portion. In contrast, Tianjin does not even impose a fine. Moreover, even if the fine is heavy because the local trading methods are still in the perfect stage, there is an unclear definition of authority and responsibility. To bring difficulties to the punishment, the enforcement power needs to be improved.

To promote the carbon emission rights trading market to play a greater role in the construction of ecological civilization, the National Federation of Industry and Commerce in the Proposal put forward four aspects of the proposal, including the establishment of carbon emissions trading supervision and management committee, comprehensive coordination, and overall decision-making. As soon as possible, the National Regulations on the Trading of Carbon Emissions Rights will be promulgated to implement the relevant supporting policies; innovate carbon financial instruments and accelerate the exploration of the market-oriented mechanism of ecological civilization; Promote the pilot construction of carbon inclusion, explore the normal mode of precision poverty alleviation, etc.

\subsection{Companies lack incentives to reduce emissions}

At present, China is in the strategic transition period of fully implementing the scientific development concept and building an environment-friendly and resource-saving society. The goals pursued by Chinese enterprises are usually focused on reducing costs, increasing production, or maximizing profits, often adopting the extensive traditional development model of high input, high consumption, and high pollution, which leads to a serious waste of resources and energy and environmental pollution. Enterprises urgently need to formulate reasonable energy conservation and emission reduction methods, properly deal with "lazy" behavior, improve existing incentive mechanisms, improve and improve corporate governance structure, and actively explore the road of transformation and development. Among them, the incentive mechanism is the basic mechanism of corporate governance, which is directly related to improving corporate governance structure and improving business performance. Under the guidance of the government's environmental protection policy, this requires the Group to formulate more policies conducive to energy conservation and emission reduction, to defuse its externalities, and to encourage power generation enterprises to actively participate in energy conservation and emission reduction work.

\subsection{The industry coverage is not comprehensive enough}

So far, other industries except the power sector have not been fully covered. The national carbon market coverage clearly eight energy-intensive industries during the "14th Five-Year Plan" period gradually included, including petrochemicals, chemicals, building materials, steel, non-ferrous, paper, electricity, and civil aviation. This means that integrating industries other than the electricity sector into the national carbon trading market has taken a step forward. The power industry is the first industry to be included in the national carbon trading market, petrochemical, chemical, building materials, steel, non-ferrous metals, paper, aviation remaining seven industries, will be "smooth docking, smooth transition" under the tone of gradually into the national carbon market, with a good carbon emission database of cement, the electrolytic aluminum industry will be given priority into the national carbon trading market.

\section{EXAMPLE: CHINA'S POWER MARKET CARBON EMISSIONS MARKET TRADING MECHANISM DESIGN}

Climate change poses a serious threat to the environment on which human beings depend. The Chinese government attaches great importance to addressing climate change and, in its national action plan, has made the establishment and operation of a carbon trading market mechanism an important response to effectively reduce carbon dioxide emissions.

\subsection{Experience in the power industry in the $E U$ carbon market}

$\mathrm{CO} 2$ emissions from the electricity sector will account for about 34\% of the country's total in 2019. From practical experience in Europe and elsewhere, introducing a carbon trading market mechanism in the power sector is one of the most effective measures to reduce carbon emissions. China has laid a solid practical foundation in recent years in the pilot of local carbon trading markets. In the country's 14th Five-Year Plan, it is imperative for the power sector to first demonstrate the mechanism for promoting carbon trading markets.

The EU is the world's oldest and by far largest carbon market, comprising $28 \mathrm{EU}$ member states and 
Iceland, Norway, and Liechtenstein, and is the cornerstone of the EU's policy on climate change. It covers the electricity sector, manufacturing, and aviation, accounting for about 45 percent of the EU's total emissions. The power sector is the largest emitter in the EU carbon market, accounting for about 31 percent of the EU's carbon market emissions in 2019 from 265 coal-fired power plants in operation and 55 percent of the total electricity market if other fossil fuels are considered.

Zhang Wei, the chief economist of the National Center for Strategic Research and International Cooperation on Climate Change of the Ministry of Ecological Environment, pointed out that the carbon market plays a key role in promoting the low-carbon development of electricity in the EU. From 2008 to 2018, the EU's renewable energy generation increased by about 1.8 times. In 2019, the EU's renewable energy generation (excluding hydropower) reached 6,500 $\mathrm{GWh}$, accounting for $35 \%$ of the EU's total electricity generation, with wind and solar generating more than coal for the first time, accounting for $18 \%$ of total electricity generation.

The first phase of the EU carbon market (20052007) and the second phase (2008-2012) due to the use of the "historical method" free allocation of most quotas, as well as the low quality of data, low-cost international offset projects and economic crisis and other factors, resulting in the EU carbon market in the first two stages of the total emission quotas lose, the carbon market has not been fully effective. Phase III (2012-2020) The EU carbon market eliminated the "bottom-up" emissions control targets proposed by member states. It adopted a series of measures to tighten the total quota and reduce the quota supply by 1.74 percent per annum from 2010 to ensure that carbon emissions levels are reduced by 21 percent by 2020 compared to 2005 levels. From 2021, the annual emission reduction factor will be further increased to 2.2 percent, reducing emissions from the carbon marketcovered industry by 43 percent compared with 2005, while the EU-wide target of 2030 emissions reductions is 40 percent. The third stage of the power companies will not receive any free quotas but will need to obtain all the quotas they need by auctioning or buying them in the secondary market.

The EU's success depends on several factors. First, the EU has a perfect top-level design, in addition to a series of supporting institutional documents, such as total set-up, quota allocation, monitoring and verification of reports, transaction performance, supervision, and other institutional documents, a good guarantee of the operation of the carbon market. In addition, the EU carbon market has experienced several problems, such as excessive quota issuance due to nonverification by enterprises' reported emissions by thirdparty verification agencies and large quota surpluses resulting from large fluctuations in the economy, based on historical production benchmarks.

Therefore, we can conclude. First, we should be moderately tight in the process of total quota setting. Second, we should combine the market quota supply and demand situation, timely adjustment of quota allocation to ensure that the carbon market price remains reasonable, to really promote power generation enterprises, and key emission reduction activities.

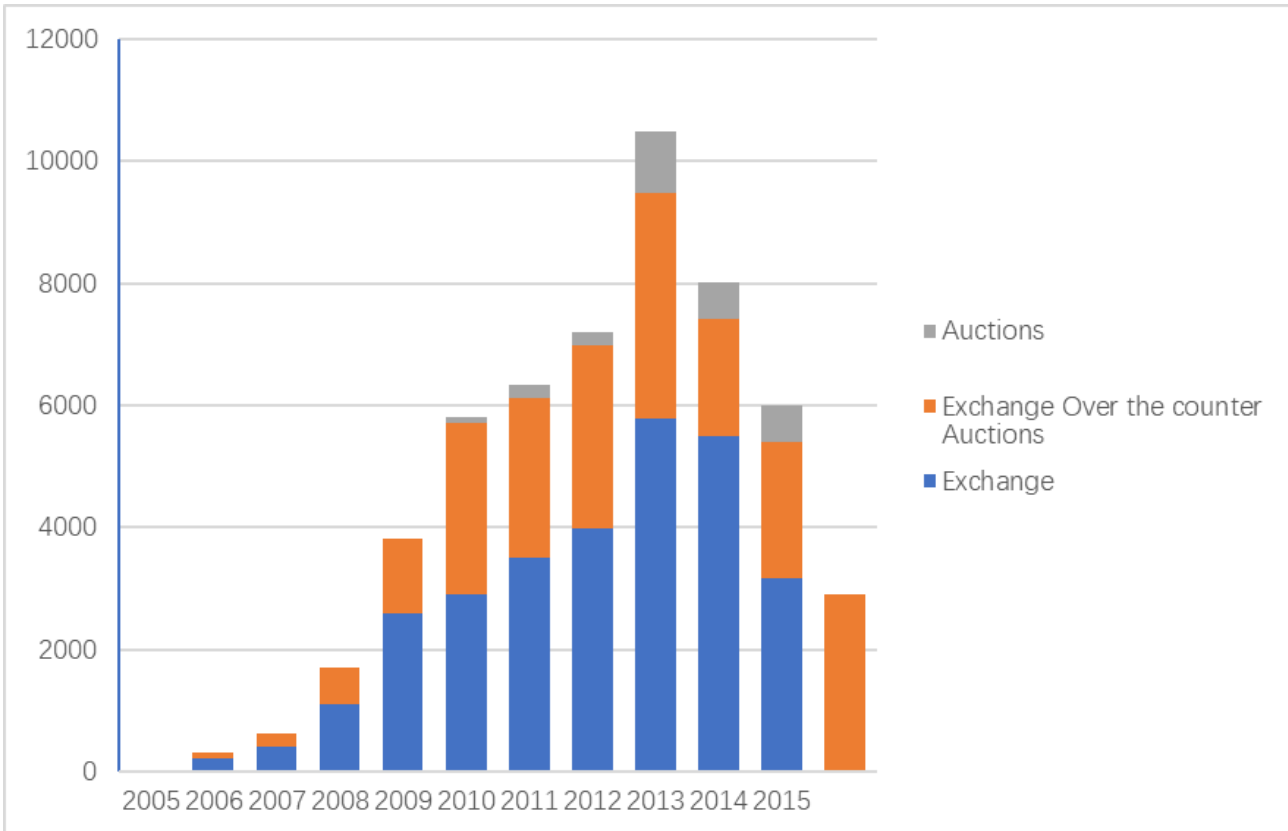

Figure 1. Distribution of trading types in EU carbon trading Market (2005-2015)

Note. Source: Brochure on the official website of the EU Carbon Trading System 


\subsection{The status quo of the carbon trading mechanism in China's electricity market}

At present, coal-fired power generation enterprises in the pilot areas of China have been included in carbon trading, Beijing, Shenzhen, Shanghai, and Fujian have also been included in the power grid enterprises, the main body is the lowest level of corporate enterprises, covering only carbon dioxide greenhouse gas. During the "14th Five-Year Plan" period, establishing the national carbon market should take the power industry as a breakthrough and gradually transition to eight key industries, including steel, chemical industry, and nonferrous. At present, the Ministry of Ecological Environment is fully soliciting the views of provincial carbon emission authorities and power enterprises and in accordance with the relevant views to further improve the quota allocation program. Since the issuance of The No. 9 and related reform supporting documents in mid2015, governments at all levels, power grid enterprises, power market operators, and relevant market entities have made significant progress and breakthroughs in the development of the electricity market around the overall goal of giving full play to the decisive role of the market in allocating resources, and have made significant progress and breakthroughs in the gradual release of electricity plans, the expansion of medium- and longterm trading scale, the promotion of spot trading pilot, and the strengthening of clean energy consumption.

However, in the process of promoting the construction of China's electric power market, many challenges are still faced. On the one hand, the construction and synergy of the power market need to be strengthened, including the coordination between medium and long-term trading and spot trading, the coordination between the inter-provincial market and the provincial market has yet to be solved. The decisive role of the market in the allocation of resources has not yet been fully developed, and a market with efficient configuration, coordinated operation, and smooth operation needs to be built. On the other hand, China's energy distribution is uneven, the reverse distribution of energy demand and resource supply is becoming more and more serious, especially clean energy difficulties. Energy structure clean transformation pressure is greater, in the power market, construction needs to fully consider clean energy, especially new energy consumption and development needs. In addition, we are changing from traditional power systems to an energy Internet, distributed power supply, energy storage, electric vehicles, and other new types of the main access to the grid. At the same time, the new generation of the power system in operation is also faced with the reduction of rotational inertia, DC, transmission end, the coupling relationship between the source network load is closer. Other new features, the development of energy Internet on the design of the power market put forward new requirements and new challenges.

To effectively meet the challenges and meet market development needs, the key is to seize the national unified power market construction this "bull nose". From the market-wide form, full power space, full architecture, full range configuration four aspects of consideration, do a good job of inter-provincial transactions and intra-provincial transactions, mediumand long-term transactions and spot trading, market transactions and grid operations, and comprehensively enhance the market's renewable energy consumption, information transparency and risk prevention and control level.

\subsection{China's carbon market trading mechanism outlook}

During the 14th Five-Year Plan period, China's response to climate change will remain unchanged. The intensity will increase when sustainable. High-quality development becomes a fundamental requirement, and the "14th Five-Year Plan" is the implementation of the Paris Agreement countries' independent contribution to the key 5 years. Therefore, for power enterprises, with low-carbon development in power planning, carbon market construction will actively promote more clean and efficient development of power enterprises. The national carbon market should give companies an optimal cost-effective way to reduce emissions. The national carbon market is a market-based emission reduction policy tool, and low or high carbon prices do not achieve cost-effective emission reductions. Therefore, the national carbon market should build a carbon price control mechanism - price corridor, the highest and lowest price. Setting price corridors does not affect trading behaviour and effectively manages market risk to ensure that carbon market emissions entities achieve emission reduction targets in a costeffective and optimized manner. Secondly, China should further improve and improve the emission data management system. Data quality is the lifeline of the carbon trading mechanism, and a perfect system of monitoring, reporting, and verification of emission data must be established. In addition, power generation enterprises as the first batch of national carbon market into the enterprise, ideologically should attach great importance to the national carbon market construction, in accordance with the new requirements of the national carbon market construction under the new situation, in action to grasp, grasp, do a good job in participating in the national carbon market preparations. Carbon trading in the power generation industry will operate steadily during the 14th Five-Year Plan period, based on which other mature energy-intensive industries (such as electrolytic aluminum, cement, and other industrial sectors) will be gradually integrated, while trading 
varieties will gradually increase, based on spot trading, other trading methods (such as futures, options, etc.) will also be usefully explored and gradually carried out. On the one hand, for the specific policies of the power generation industry, it is expected that the national benchmark value for coal-fired power generation units will be tightened year by year. However, the allocation of quotas is still mainly in the form of free distribution. Still, it will gradually introduce auction methods, so carbon emissions to coal-fired power generation enterprises to bring greater cost pressure. The incentive policy may also be phased out for gas units after a transition period of 1-2 years, i.e., gas units will also be required to perform mandatorily. In this issue of Sharon, experts believe that the "14th Five-Year Plan" is China's socio-economic, and even the development of the energy industry, the key period, and the power industry's carbon trading market is an arrow in the string, it is imperative, other industries should also be prepared. The establishment of a carbon market mechanism is conducive to promoting energy conservation and emission reduction and coal power peak as soon as possible, developing more clean and renewable energy power.

\section{CONCLUSIONS}

At present, China's electricity market carbon trading trial is progressing smoothly, but there are still problems of uncoordinated construction and coordination of the power market and uneven energy distribution. In the future, the key is to seize the national unified power market construction this "bull nose", from the marketwide form, full power space, full architecture, full range of configuration four aspects of consideration. Do a good job of inter-provincial and intra-provincial transactions, medium- and long-term transactions and spot trading, market transactions and grid operations, comprehensively enhance the market's renewable energy consumption, information transparency, risk prevention, and control level.

\section{REFERENCES}

[1] Ding Ding, Pan Fang.On the legal properties of carbon emissions rights [J].Law Journal.(2012)

[2] Yanping Su.On the legal properties of carbon emissions rights [J].Journal of Shanghai Institute of Finance.(2012)

[3] Hui Zhang.Carbon Trading Policy Design and Market Performance Research [D].Nanjing University.(2017)

[4] Sun Ren.Analysis of Carbon Rights in the View of Law Economics [J].Environment and Development.(2020)
[5] Yushan Ji,Tong Li.Countermeasures on establishing China [J].Economic aspect.(2012)

[6] Min Rao.Research on the Internal Mechanism and Countermeasures of Building China Carbon Emission Trading Market [D].Central South University for Nationalities.(2013)

[7] Junrong Zhang, Zidan Wang, Ling Tang, Lean Yu.Study on Beijing-Hebei Carbon Emission Trading Policy Based on System Dynamics [J].China Management Science.(2016)

[8] Sheng Huang, Xingguo Li .Hebei carbon emissions and carbon trading in Hebei province under the threshold [J].Journal of Tsinghua University (Natural Science Edition).(2017)

[9] LihongLi , BowenYang .Study on Legislative Coordination Mechanism of Regional Carbon emission Trading in Beijing-Tianjin-Hebei Region [J].Hebei Law.(2016)

[10] Zhao Changle.Study on Carbon emission Trading Mechanism in China [D].Wuhan University of Engineering.(2018)

[11] Chinese Certified Emission Reduction (CCER) refers to the voluntary emission reduction of greenhouse gases that has been recorded and registered in the national registration system in accordance with the Interim Measures for the Administration of Voluntary Greenhouse Gas emission Reduction Transactions issued and implemented by the National Development and Reform Commission. 